\title{
La teoría crítica feminista como correctivo epistémico en
}

psicología

\author{
The feminist critical theory as an epistemic corrective in
}

psychology

S. García Dauder

Recibido: 03/12/2018

Aceptado: 02/07/2019

\section{RESUMEN}

El artículo comienza analizando la situación de las mujeres en la psicología (como sujetos y objetos de conocimiento); para posteriormente abordar cómo el feminismo, como teoría crítica y movimiento social, ha transformado la psicología. En primer lugar, describe cómo las pioneras psicólogas utilizaron sus conocimientos científicos para desmontar mitos sobre la inferioridad de las mujeres y los efectos de la educación en su salud. En segundo lugar, analiza cómo los feminismos de los 70 denunciaron el androcentrismo en salud mental y crearon los grupos de auto-conciencia como formas colectivas de politizar los malestares con efectos terapéuticos. Por último, cómo las psicólogas feministas han criticado la esencialización de las diferencias y los dualismos de sexo/género y la patologización de la diversidad. El artículo termina con una reflexión sobre las relaciones entre el feminismo y la psicología hoy.

Palabras clave: Psicología, feminismo, mujeres, salud mental, epistemología.

\section{ABSTRACT}

The article begins by analyzing the situation of women in psychology (as subjects and objects of knowledge); to later address how feminism, as a critical theory and social movement, has transformed psychology. First, it describes how the pioneering psychologists used their scientific knowledge to dismantle myths about the inferiority of women and the effects of education on their health. Second, it analyzes how the feminisms of the $70 \mathrm{~s}$ denounced androcentrism in mental health and created the groups of self-consciousness as collective forms of politicizing discomforts with therapeutic effects. Finally, how feminist psychologists have criticized the essentialization of sex and gender differences and dualisms and the pathologization of diversity. The article ends with a reflection on the relations between feminism and psychology today.

Keywords: Psychology, feminism, women, mental health, epistemology.

S. García Dauder es docente titular de Psicología Social en la Universidad Rey Juan Carlos (Madrid). Correo electrónico: dau.dauder@urjc.es. ID: https: / / orcid.org/0000-0002-7978-5263

Cómo citar este artículo: García Dauder, S. (2019). La teoría crítica como correctivo epistémico en psicología. Atlánticas. Revista Internacional de Estudios Feministas, 4 (1), 117-150. doi: http: / / dx.doi.org/10.17979/ arief.2019.4.1.4843 


\section{LA EXCLUSIÓN HISTÓRICA DE LAS MUJERES COMO SUJETO DE CONOCIMIENTO EN PSICOLOGÍA}

En su ya clásico libro Ciencia y Feminismo, Sandra Harding (1996) proponía el análisis, para cada disciplina, de la situación de las mujeres en las ciencias (como sujetos y como objetos de conocimiento), a la situación de la ciencia en el feminismo (las implicaciones epistemológicas de la presencia o ausencia de mujeres en una disciplina y hasta qué punto el feminismo como teoría crítica y movimiento social ha contribuido a mejorar la ciencia). En su libro más reciente, Diversity \& Objectivity, Harding (2015) va más allá relacionando la diversidad de posiciones de sujeto, múltiples y contradictorias, en una comunidad científica con una mayor objetividad; y su reverso, asocia la homogeneidad del sujeto de conocimiento a campos de ignorancia y ciencia sin hacer derivados de valores hegemónicos incuestionados.

A lo largo de la historia de la psicología podemos identificar sistemas de opresión o "violencias epistémicas" que favorecen a grupos privilegiados, mientras construyen identidades en desventaja epistémica. Las mujeres como colectivo, junto con otros grupos, han sido desacreditadas como autoridades cognitivas, construidas como no conocedoras, al tiempo que se ha invisibilizado su resistencia, transformándola estratégicamente en ignorancia o incompetencia (Tuana, 2006). La historia de la psicología está narrada desde el relato repetido de mentes masculinas que conocen naturalezas o psicologías femeninas. Es decir, las mujeres aparecen excluidas como sujetos de conocimiento al tiempo que son construidas como objetos de estudio. La pregunta que surge es si esta homogeneización del sujeto de la psicología -en términos masculinos y de blanquitud- es epistemológicamente relevante. Es decir, si frente a la visión del psicólogo como científico neutro, objetivo y libre de valores, dicha posición de sujeto homogénea ha influido en las formas de producción de conocimiento psicológico, especialmente cuando se ubican como objetos de estudio las diferencias entre los sexos o las raciales.

Pero la historiografía feminista nos ha desvelado un "secreto muy bien guardado" por la historia de la psicología: la presencia de pioneras psicólogas en los orígenes de la disciplina (Scarborough y Furumoto, 1987). Y una 
ignorancia consolidada en los manuales: el olvido de mujeres psicólogas presentado falsamente como ausencia (Bohan, 1992). Para Gerda Lerner (1992), es necesario recuperar los nombres de las pioneras y sus contribuciones en una suerte de proyecto compensatorio. Por ejemplo, que en los salones de grados estén los cuadros de Mary Calkins o Margaret Washburn, ambas presidentas de la APA (Asociación Estadounidense de Psicología) en 1905 y 1921 respectivamente. Pero también que se incluyan en los manuales los nombres y las contribuciones de otras muchas pioneras, reconocidas por sus compañeros en la época y que hicieron contribuciones importantes (en las principales revistas, congresos, etc.); todo ello a pesar de unas condiciones sociales de segregación sexual y de múltiples discriminaciones (Scarborough y Furumoto, 1987; García Dauder, 2005).

Pero para Lerner este proyecto compensatorio no es suficiente. Es preciso narrar la historia "en sus propios términos", her-story: hasta qué punto las condiciones de producción de conocimiento fueron desiguales para las mujeres psicólogas respecto de sus compañeros de disciplina y cómo afectó a sus formas de hacer psicología. Ello implica romper con el mito de la meritocracia en ciencia y visibilizar los diferentes mecanismos de discriminación y exclusión a los que se enfrentaron las pioneras por el hecho de ser mujeres (ideológicos, institucionales o subjetivos). Los más evidentes: que las universidades se resistían a admitirlas como estudiantes de pleno derecho y la construcción de la mujer científica como una "contradicción en sus propios términos" (Rossier, 1992). Así, no solo es preciso denunciar la producción de ignorancia histórica en psicología, obviando los nombres y contribuciones de las mujeres y de otros colectivos excluidos, sino también recuperar la memoria de sus resistencias y el conocimiento producido desde dichas posiciones.

\footnotetext{
- No solo recuperar las psicólogas estadounidenses, también las pioneras en otros contextos. Ver por ejemplo, García Colmenares (2011) para conocer las primeras psicólogas españolas, Winkler (2007) para recuperar la historia de las "pioneras sin monumentos" en Chile y Argentina, o Guevara Ruiseñor (2015) para rescatar las psicólogas de México, Cuba, Brasil o Colombia.
} 
La "historia en sus propios términos" ha recuperado ejemplos de cómo la presencia de mujeres en psicología, como grupo social excluido, permitió la visibilización de lo no cuestionado, ampliando el horizonte cognitivo de una comunidad masculina ciega y acrítica ante determinados campos de ignorancia. En ese sentido, su presencia sin garantías (necesaria pero no suficiente) ayudó a transformar la disciplina, haciéndola más objetiva y justa socialmente, especialmente cuando se producía conocimiento psicológico sobre las mujeres o las diferencias sexuales. Nancy Tuana (2006) lo ha descrito como el paso de "las epistemologías de la ignorancia a las epistemologías de la resistencia".

Para Harding (2015: 18), "ciencia y sociedad se co-producen mutuamente"; esto es, sociedades sexistas y racistas tenderán a promover ciencias sexistas y racistas (aunque se planteen como objetivas y libres de valores e intereses). Por ello, "la norma social de la diversidad y la norma epistémica de la objetividad pueden apoyarse mutuamente" (2015: 23) y promover conocimiento y justicia social. Según esta autora, la investigación que parte del punto de vista de colectivos externos a las comunidades científicas dominantes (es decir, cuyo punto de partida es las vida cotidiana de grupos oprimidos) puede maximizar la objetividad. Por ejemplo, las historiadoras feministas de la ciencia nos han enseñado cómo los movimientos sociales han tendido a mejorar la ciencia gracias a las críticas que dirigen hacia los planteamientos hegemónicos, dando lugar a visiones menos distorsionadas y parciales. No es solo una cuestión de presencia de mujeres (o de miembros de otros colectivos excluidos), sino de asegurar que la disciplina "respeta los valores e intereses de todos los ciudadanos al tiempo que protege los grupos más vulnerables política y económicamente" (2015: xi). Por ello, la búsqueda de conocimiento requiere de políticas democráticas y participativas:

“si éste no fuera el caso, solo las élites de género, raza, sexualidad y clase que predominan en las instituciones de búsqueda de conocimiento, tendrán la oportunidad de decidir cómo plantear sus preguntas de investigación, y tenemos suficientes razones para sospechar de la 
localización histórica desde donde tales preguntas serán de hecho planteadas" (Harding, 1991: 124).

Para apoyar el planteamiento de Harding con el ejemplo de la psicología, analizamos en este artículo hasta qué punto el feminismo como movimiento social y teoría crítica ha funcionado como correctivo epistémico en psicología, desvelando campos de ignorancia e identificado ciencia sin hacer (planteando otras preguntas, marcos teóricos, métodos e interpretaciones...). Describimos el paso "de la psicología construye a la mujer y lo femenino al feminismo reconstruye y transforma la psicología" (Kitzinger, 1993), desde esas epistemologías de resistencia que señala Tuana (2006). En concreto, nos vamos a centrar en el conocimiento feminista que las primeras psicólogas produjeron sobre la educación de las mujeres y su relación con la salud, desmontando mitos sobre las diferencias sexuales y la inferioridad mental de las mujeres; las críticas y transformaciones que las psicólogas feministas aportaron a la disciplina en los años 70, especialmente denunciando el modelo androcéntrico de salud mental y, como consecuencia, el sexismo en teorías y prácticas clínicas; y, por último, cómo los movimientos por la diversidad sexual también han funcionado como correctivos epistémicos, despatologizando la diversidad y planteando re-conceptualizaciones de conceptos psicológicos como la identidad de género o la masculinidad y la feminidad.

\section{EL CONOCIMIENTO CIENTÍFICO DE LAS PIONERAS PSICÓLOGAS COMO RESISTENCIA FEMINISTA}

Frente al mito de la objetividad y la meritocracia en la ciencia, las historiadoras no solo han recuperado los nombres y contribuciones de las primeras psicólogas, también las condiciones sociales y las desigualdades de género que limitaron e influyeron en sus carreras y práctica científica. Dentro de dichas experiencias diferenciales de género, han visibilizado tanto los mecanismos de exclusión como sus estrategias de resistencia. Por ejemplo, han recuperado su feminismo científico mediante el cual usaron la psicología para desmontar los mitos existentes sobre las diferencias mentales entre los sexos y la inferioridad "natural" de las mujeres (Scarborough y Furumoto, 1987). 
Respecto a la exclusión institucional que experimentaron las pioneras, pocos manuales de historia -por no decir ninguno- mencionan que las principales universidades prohibían la presencia de mujeres en sus aulas, que entraban de forma excepcional como "estudiantes especiales" u oyentes, que no reconocían sus doctorados o que ponían todo tipo de trabas para que pudieran desarrollar su carrera profesional desde la academia, sobre todo si se casaban (Scarborough y Furumoto, 1987). Como consecuencia, se dio una segregación horizontal en la disciplina: una psicología "pura" desde dentro de la academia, masculinizada y legitimada; y una psicología "aplicada" desde los ámbitos de reforma, feminizada, desprestigiada y excluida de los mecanismos de reconocimiento oficial (García-Dauder, 2005). Desde esta última, solían detectar e investigar problemas sociales para su transformación. Por ejemplo, toda la producción de conocimiento aplicado e interdisciplinar de la llamada Escuela de Chicago de Mujeres sobre las consecuencias de la explotación laboral de las mujeres (y la necesidad de una regulación en términos de horarios y carga de trabajo), así como sus investigaciones sobre las condiciones laborales de determinados entornos, como los talleres de la confección o las fábricas, ejerciendo como inspectoras de salud laboral (García Dauder, 2010).

Estos mecanismos institucionales de exclusión, mediante los cuales se excluía a las mujeres de la academia y eran conducidas a actividades de reforma, estuvieron apoyados por otros mecanismos discursivos e ideológicos. Los principales discursos de la época recordaban constantemente a las mujeres las inconveniencias de la educación superior y el ejercicio de una carrera profesional. Se pasó del no pueden las mujeres estudiar (por cráneos más pequeños o cerebros de menor peso), al no deben, ya que su presencia en las aulas desmontaba el argumento de la incapacidad (García Dauder, 2005). Se podría decir que dichos argumentos eran comunes en los "intelectuales" de diferentes países, al igual que lo fueron las resistencias de las pioneras. En el contexto español, por ejemplo, las tesis sobre la inferioridad mental de las mujeres y los nocivos efectos de la educación en la salud de las mujeres, fueron rebatidos por teóricas como Concepción Arenal o Emilia Pardo Bazán en los Congresos Pedagógicos. No es casual que las dos primeras doctoras en Medicina en España, Dolores Aleu Riera y Martina Castells, trataran de 
demostrar las bondades de la educación para las mujeres, en su salud y en su moral (Flecha, 1996; Nogueiras, 2018). Lo mismo harían otras médicas pioneras en diferentes países, como Elizabeth Garret Anderson en Inglaterra o Mari Putnam Jacobi en EEUU (Vertinsky, 1994).

En el ámbito de la psicología, autores de re-nombre como Stanley Hall, James Cattell, Edward Titchener o Edward Thorndike, entre otros, perdían su objetividad y cientificidad cuando de educación para las mujeres se trataba. Los argumentos se centraron en la hipótesis de la "inversión útero-cerebro": la fecundidad intelectual acarrea esterilidad corporal y el consecuente suicidio de la raza (tesis defendida por Cattell o Hall). O bien la tesis, vía Darwin, de la menor variabilidad de las mujeres: las mujeres son menos variables, más mediocres, que los varones, por eso no hay mujeres genios y por eso es un error invertir en ellas en educación superior (tesis defendida por Thorndike por ejemplo). Helen Thompson Woolley (1910: 342), brillante pionera psicóloga, describió muy bien estos argumentos:

\begin{abstract}
"Aquellos que se sienten contrarios a permitir que las mujeres tengan una plena oportunidad de desarrollo mental han cambiado el énfasis de su argumento desde un punto de vista personal a uno social. La queja ya no es que la mujer se perjudicará a sí misma por el excesivo esfuerzo mental y físico que supone la formación intelectual superior, sino que perjudicará a la sociedad reduciendo su propia actividad reproductiva (matrimonios tardíos, menores tasas de matrimonios, menor número de hijos, oposición entre las funciones intelectuales y sexuales) [...]. La conclusión parece ser que el deber superior de la mujer es frenar voluntariamente el desarrollo de sus propias capacidades mentales ante la amenaza de perjudicar a la sociedad -una forma de ascetismo un tanto difícil de suscribir".
\end{abstract}

En 1882, un grupo de licenciadas en diferentes universidades de EEUU (entre las que se encontraban psicólogas como Christine Ladd-Franklin) crearon en Boston la Association of Collegiate Alumnae (ACA), más tarde convertida en la Amercian Association of University Women (AAUW). Este grupo de presión pionero tuvo como objetivo la promoción de la educación universitaria en 
mujeres (Rossiter, 1992). Urgía un estudio empírico que desmintiera estas afirmaciones que socavaban la motivación de las universitarias. El mismo año de su creación, la ACA construyó un cuestionario que envió a 1290 licenciadas y miembros de la asociación. Los resultados: el 78\% gozaba de muy buena salud y solo el 17\% tenía problemas. Las enfermedades y los desórdenes nerviosos de este último grupo no se explicaban en el informe por una incompatibilidad útero-cerebro, sino por la ignorancia en determinados temas sanitarios y por las mórbidas consecuencias de determinadas expectativas y tradiciones culturales sobre las mujeres. El informe de la ACA fue publicado en un primer momento por el Massachusetts Bureau of Statistics of Labor en 1885, bajo el título Health Statistics of Women College Graduates. Si bien pocos lectores tuvieron acceso a esta primera publicación -y la difusión del informe era uno de los principales objetivos de la ACA-, al año siguiente John Dewey lo dio a conocer con una reseña en el Popular Science Monthly, con el título "Health and sex in higher education", donde comentaba de forma extensa sus hallazgos (Rosenberg, 1982).

Las psicólogas pioneras criticaron el sexismo de las teorías científicas que esencializaba las diferencias entre los sexos como naturales y justificaba la inferioridad mental de las mujeres. No es casual que fuera una psicóloga, Leta Stetter Hollingworth, quien llevara a cabo una tesis doctoral, Functional Periodicity, para desmontar el mito de la disminución del rendimiento mental y motor de las mujeres durante la menstruación.

"Varones de intereses y profesiones de lo más diverso han escrito sobre este tema -historiadores, médicos, abogados, filósofos, psicólogos, novelistas y educadores. Varones a los que nunca se les hubiera ocurrido escribir con autoridad sobre ningún otro tema sobre el cual no poseyeran un conocimiento seguro y experto, no han dudado en realizar las más categóricas afirmaciones respecto a las habilidades mentales y motoras de las mujeres durante el período menstrual" (Hollingworh, 1914: 97).

Como ella misma señalaba, anticipando las epistemologías del punto de vista y la importancia de mujeres investigadoras como conocedoras encarnadas: 
"Es un tema sobre el cual nada definitivo puede saberse en la medida en que las únicas que pueden realmente conocerlo, las propias mujeres, han aportado un escaso testimonio (...) Así, con el tiempo, podrá ser escrita una psicología de la mujer basada en la verdad, y no en la opinión; sobre evidencia precisa y no anecdótica; sobre datos rigurosos y no sobre secuelas de pensamiento mágico" (Hollingworth, 1914: 99).

Tampoco es casual que ella misma dedicara varios de sus trabajos a desmontar la hipótesis de la inferior variabilidad física y mental de las mujeres o la naturalización del instinto maternal (García Dauder, 2005). La presencia de mujeres en Medicina provocó que se cumpliera el deseo de Hollingworth. A finales del XIX y principios del XX el "laboratorio feminista" se puso a trabajar para desmontar gran parte de los mitos sobre la "incapacidad menstrual" de las mujeres. Destaca la tesis doctoral de Mary Jacoby The question of rest for women during menstruation o los trabajos de Clelia Mosher, como "Normal menstruation and some of the factors modifying it" (Vertinsky, 1994).

Dentro de esas epistemologías de la resistencia, destaca la "lucidez social" de las pioneras en las controversias sobre diferencias sexuales en procesos cognitivos y su sensatez al señalar a sus compañeros la imposibilidad de encontrar diferencias sexuales "naturales"; o lo que era lo mismo, la imposibilidad del "experimento perfecto" que anulara toda influencia social (Morawski, 1988). Retomando las palabras de la que fuera doble presidenta de la Asociación Estadounidense de Psicología y de Filosofía, Mary Whiton Calkins (1896: 430):

Mr. Havelock Ellis (...) y Dr. Jastrow, quizá, con la expresión 'rasgos mentales masculinos y femeninos', pretenden una distinción entre el intelecto per se masculino y femenino. A mi entender se trata de algo fútil e inviable, debido a nuestra total incapacidad para eliminar los efectos del ambiente. Hoy en día las diferencias en la educación y tradición de varones y mujeres comienzan en los meses más tempranos de la infancia y continúan a lo largo de la vida. La mayor parte de las preferencias que han sido encontradas en los dos experimentos, por 
ejemplo la preferencia de las mujeres por lo que está relacionado con la casa, son obviamente intereses cultivados.

Esta psicología relacional y social se mostraba de forma recurrente en las primeras psicólogas, en palabras de Amy Tanner: "las tendencias reales de las mujeres no podrán ser conocidas hasta que sean libres de elegir, no más que las de un perro encadenado" (1896: 550). Frente al sexismo y la exageración de las diferencias, dedicaron sus esfuerzos de investigación experimental al estudio de las semejanzas entre los sexos. Helen Thompson Woolley, por ejemplo, hizo su tesis doctoral sobre las semejanzas en rasgos mentales, The Mental Traits of Sex. De ella, concluyó: "Si realmente hubiera una diferencia esencial de instintos y características que determinara las diferencias (...) no sería necesario malgastar tanto esfuerzo en conseguir que chicos y chicas siguieran las líneas de conducta propias de cada sexo" (Woolley, 1903: 181).

A los mecanismos institucionales e ideológicos de exclusión, se le sumaban las barreras subjetivas interiorizadas por las propias mujeres: el dilema matrimonio versus carrera, las demandas del "imperativo familiar" y las negociaciones identitarias entre su identidad de género y su identidad profesional. Como ha señalado Margaret Rossiter (1992), la mujer científica se había construido como una "contradicción en sus propios términos": ser mujer y responder a las normas y valores asociados con lo femenino, y ser científica y responder a las masculinizadas normas y valores de la ciencia (objetividad, control y dominio). Como mujeres, eran poco científicas; como científicas, poco mujeres.

Pero también las psicólogas supieron sacar rendimiento teórico de estos conflictos subjetivos. En 1929, la psicoanalista francesa Joan Rivière escribió “La feminidad como mascarada", un artículo donde analizaba las estrategias de las mujeres que se introducían en el mundo "masculino" de la academia: "intentaré mostrar que las mujeres que desean la masculinidad tienden a ponerse una máscara de feminidad para evitar la ansiedad y la temida condena de los varones" (Riviere, 1929: 35). Otra psicóloga social, Jessie Taft (1916), discípula de George H. Mead, utilizó el interaccionismo simbólico para explicar en su tesis doctoral el surgimiento del movimiento de la mujer como producto del 
conflicto subjetivo de las mujeres de la época, entre sus aspiraciones y las restricciones que les imponía la sociedad. Taft citaba y recogía el trabajo de la socióloga y escritora Charlotte Perkins que en El empapelado amarillo de 1892 ya había descrito la ceguera de los médicos ante los conflictos subjetivos de las mujeres y sus "curas de reposo". Como han señalado Ehrenreich y English (1990), hubo una auténtica "epidemia de histeria" en el siglo XIX. La mujer histérica estaba a un paso de la mujer ideal romántica: un ser que debía ser frágil, dependiente, pasivo, sin deseo sexual, doméstico y ocioso. Algunas de las pioneras, como Perkins o Jane Addams tuvieron sus propias crisis, interpretadas por ellas mismas como protesta desesperada ante las ataduras del rol femenino victoriano. En su caso, como describió Taft en su tesis, transformaron la histeria en militancia (a través de la escritura o la reforma respectivamente).

En definitiva, es de "justicia epistémica" reivindicar el papel no reconocido de las pioneras en el desarrollo de una psicología más rigurosa, su énfasis en el papel de lo social en la explicación de las diferencias sexuales, así como en los conflictos subjetivos de las mujeres de la época; su apuesta por la interdisciplinariedad y sus contribuciones de transformación social desde ámbitos aplicados o de reforma (Scarborough y Furumoto, 1987; García Dauder, 2005). Ante las ofensas personales de sus propios compañeros de disciplina y los desesperantes obstáculos institucionales, las primeras psicólogas sintieron la responsabilidad de utilizar sus conocimientos y su posición como científicas para mejorar la situación de las mujeres. De esta forma, las pioneras contribuyeron al desplazamiento de la "psicología construye lo femenino y la mujer" al "feminismo reconstruye a la psicología" (Kitzinger, 1993).

\section{DE LA PSICOLOGÍA CONSTRUYE LA MUJER/LO FEMENINO, AL FEMINISMO RECONTRUYE LA PSICOLOGÍA}

Los análisis feministas de la psicología y del conocimiento psicológico también han experimentado la transición descrita por Harding de "la situación de las mujeres en la ciencia" a la "situación de la ciencia en el feminismo". En las décadas conservadoras de los 50-60 se produjeron denuncias aisladas sobre la 
situación desigual de las mujeres en la psicología, así como de actitudes y prácticas sexistas en la academia. Pero no será hasta la década de los 70, coincidiendo con la "segunda ola" del feminismo y la fundación de organizaciones como la National Organitation of Women (NOW) en 1966, que las mujeres psicólogas adquirieron conciencia de grupo en situación de desigualdad. Impulsadas por los movimientos de nueva izquierda, estudiantiles, de anti-psiquiatría y contraculturales -y a su vez en respuesta al sexismo de estos grupos-, se organizaron para denunciar su situación y promover cambios tanto reformistas como radicales. Entre los primeros, llevaron a cabo investigaciones de denuncia sobre su discriminación en la academia para promover cambios legales. Entre los segundos, impulsaron "grupos de concienciación" informales para la reflexión política sobre su situación y realizaron (siguiendo la estela de las pioneras) análisis críticos sobre el sexismo y el androcentrismo en la disciplina.

Del Congreso anual de la APA de 1969, y de las relaciones y charlas informales entre activistas feministas durante dicho congreso, convertidas en "grupos de concienciación", se fundó la Association for Women Psychologists (AWP) (Unger, 1998). La AWP derivó en una asociación informal externa a la APA, extra-académica, no jerárquica y de carácter más radical y activista que la posterior División 35 de "Psicología de las Mujeres". Esta última surgió de forma paralela, y con carácter formal y académico, como producto de la creación de una comisión de investigación desde dentro de la APA -el Committee for Women in Psychology (CWP) dirigido por Martha Mednickcon el objetivo de promocionar el estudio sobre la situación de las mujeres en la psicología como sujetos y como objetos de conocimiento. Son años que coinciden "curiosamente" con la elección de la tercera y cuarta presidentas de la APA, Ann Anastasi en 1972 y Leona Tyler en 1973, rompiendo con una ausencia de mujeres presidentas de más de 50 años.

Por otro lado, en respuesta a la deficiente aceptación de los estudios sobre psicología de las mujeres, concebidos por las revistas main/male stream como "particulares o minoritarios", se fundaron las revistas Sex Roles en 1975 y 
Psychology of Women Quarterly en 1977, cuya línea editorial se dirigía fundamentalmente a estudios empíricos sobre "psicología de las mujeres" y "psicología del género o de las diferencias sexuales". A partir de la década de los 90, los diferentes desarrollos de las epistemologías feministas junto con la emergencia del socioconstruccionismo en psicología social, posibilitaron una "psicología feminista" que entendía la objetividad como conocimientos situados, reflexivos y responsables, y las críticas políticas y epistemológicas como indisociables (Morawski, 1997). Así, no es hasta 1991 que se crea una revista que integra las palabras "psicología" y "feminismo": Feminism $\mathcal{E}$ Psychology.

Al igual que ocurrió con las pioneras psicólogas, en la década de los 70 el punto de vista feminista transformó la disciplina y actuó como correctivo epistémico. Los análisis feministas de la psicología fueron críticos con los esencialismos biológicos, y desde análisis psicosociales enfatizaron la importancia del contexto social en el estudio de las diferencias sexuales (Unger, 1998). En el campo de la terapia, las psicólogas feministas denunciaron el modelo androcéntrico de salud mental y la existencia de dobles estándares en la evaluación clínica y los tratamientos.

Ellen Herman (1995) ha analizado el "curioso cortejo" de la psicología y el feminismo durante esta década. "A los ojos de muchas feministas, la psicología era poco más que sexismo disfrazado de ciencia” (1995:279). Como ha analizado Erica Burman (1998) en su libro La deconstrucción de la psicología evolutiva, las feministas tenían buenos motivos para recelar de los expertos psicólogos que tras la guerra habían culpabilizado a las madres especialmente a las trabajadoras- tanto de "neurosis de soldados" como de desastres sociales (las investigaciones sobre el apego funcionaron como un excelente control social). Pero, como ha señalado Herman, si bien la psicología ayudó a "construir la feminidad", también -y en respuesta a ello- provocó en parte la nueva ola del feminismo que, a su vez, se valió de conceptos psicológicos que ayudaban a explicar los aspectos subjetivos -no solo los materiales- de la opresión patriarcal. Esta convergencia de "la psicología 
construye la feminidad" y "la psicología construye a la feminista" se hizo patente muy especialmente en la obra de feministas y analistas psicosociales como Naomi Weisstein, Betty Friedan, Kate Millett, Sulamith Firestone, Philip Chesler, entre otras.

En 1968, Naomi Weisstein publicó su polémico “Kinder, küche, kirche as scientific law: Psychology constructs the female" ${ }^{2}$. "El argumento central de mi artículo es el siguiente. La psicología no tiene nada que decir sobre cómo son las mujeres, lo que necesitan o lo que quieren, especialmente porque la psicología no lo sabe" (Weisstein, 1968:197). Y no lo sabe, en opinión de Weisstein, por su obsesión por los rasgos internos y su descuido del contexto social. Para explicar el comportamiento de las mujeres es necesario comprender las condiciones y expectativas sociales bajo las cuales viven las mujeres. Weisstein acudía a los experimentos clásicos en psicología social presentados en oposición a las teorías biologicistas- para demostrar la necesidad de analizar las influencias de la situación en el comportamiento de las personas. En una línea similar, en 1979 la psicóloga social Carolyn Sherif escribió "What every intelligent person should know about psychology and women" y "Ethnocentrism, androcentism, and sexist bias in psychology", donde de forma irónica nos presentaba su "breve curso sobre cómo perpetuar un mito social" sobre diferencias sexuales. Del mismo año es otro artículo clásico de Rhoda Unger, "Toward a redefinition of sex and gender", donde introdujo por primera vez el concepto "género" en una revista como la American Psychologist, advirtiendo que centrarse en las diferencias sexuales oscurecía el análisis sobre las semejanzas y sobre los determinantes situacionales de la conducta (1979: 1090) ${ }^{3}$. Todos ellos, artículos que ejemplifican el énfasis de las psicólogas sociales feministas del momento no solo en el estudio de estereotipos y prejuicios de género, también en el análisis del poder y la influencia del contexto social (Unger, 1998).

2Feminism \& Psychology ha dedicado un monográfico especial a dicho texto en su volumen 3(2) de 1993.
${ }^{3}$ Feminism \& Psychology ha dedicado un monográfico especial a dicho texto en su volumen 17(4) de 2007. 
Betty Friedan y la Mística de la feminidad (1963) puede considerarse otro ejemplo de la "psicología construye la feminista". Formada en psicología humanista, Friedan conceptualizó el "problema que no tiene nombre", antecedente teórico de los "malestares de género" en mujeres blancas de clase media. Licenciada en psicología de forma brillante, Betty Friedan había abandonado sus estudios de doctorado en Berkeley para dedicarse a una vida doméstica. Tras una investigación que le encargó el Smith College sobre las contribuciones de las mujeres a la ciencia, comenzó a tomar conciencia de la pérdida colectiva de identidad de las mujeres de su generación. En La Mística de la Feminidad documentaba y denunciaba la situación de muchas mujeres blancas de clase media con estudios superiores que, como ella misma, habían sido convertidas por la sociedad en "amas de casa" sin proyectos propios. "A las mujeres no se las tomaba en serio, y tampoco las mujeres se tomaban en serio a sí mismas" (Friedan, 1963). El libro tuvo gran impacto y muchas mujeres se identificaron con las vidas vacías de mujeres educadas que abandonaban y sacrificaban sus proyectos individuales para dedicarse a la vida de los demás. No obstante, también fue criticado por ser excesivamente psicológico y feministas negras y lesbianas matizaron que la privilegiada situación de mujeres blancas heterosexuales de clase media no se podía generalizar a todas las mujeres (hooks, 1984).

Kate Millett representa también la confluencia de la teoría feminista con la psicología social. Su libro Política Sexual (Millett, 1969) es un clásico del feminismo radical, al conceptualizar el patriarcado como "política sexual" y las relaciones entre los sexos como relaciones de poder que demandan cambios no solo públicos sino también privados. En dicho libro utilizó el concepto de "identidad", la dimensión social de la experiencia subjetiva, y lo asoció a los procesos de socialización de género como base ideológica del poder patriarcal. Recogiendo el lema de Simone de Beauvoir "la mujer no nace se hace" y el concepto de "identidad genérica esencial" de Robert Stoller, Millett enfatizó la construcción social del sexo y de la sexualidad en un momento de auge sociobiológico. Junto a ello, criticó los escasos trabajos desde la psicología sobre las repercusiones psíquicas de la dominación masculina y, al igual que Beauvoir, Friedan, Sulamith Firestone, Eva Figes o Germaine 
Greer, dedicó no pocas críticas a la ideología sexista y androcéntrica del psicoanálisis.

No obstante, las aportaciones feministas de Millett en relación con la psicología vinieron también de su asociación con movimientos de antipsiquiatría y de su propia experiencia de internamiento en un psiquiátrico obligada por su familia- en la década de los 70 tras reconocer públicamente su lesbianismo. Las críticas de Millett a la psicología y psiquiatría clínica fueron reflejadas en sus autobiografías En pleno vuelo (1974) y en The Looney Bin-Trip (1990). No es casual que Sulamith Firestone, otra de las "grandes" feministas radicales, acudiera al relato biográfico también para describir su experiencia en un psiquiátrico (tampoco que se convirtiera en una "artista habituada al filo existencial" tras su retirada del activismo, como lo hizo Millett). Tras la Dialéctica del sexo, donde sostenía que el objetivo de la revolución feminista no debía ser la eliminación de privilegios masculinos, sino la distinción sexual en sí misma, solo escribió otro libro, Airless Spaces. En relatos a modo de viñetas, recogía la dura confluencia del encierro psiquiátrico, las crisis, la vida en la calle y el abandono material de las feministas radicales, como fue su caso, el de Millett o el de Valerie Solanas (a quien le dedica un capítulo).

Algunas autoras han interpretado la histeria como expresión paradigmática de lucha feminista y a las "histéricas" como heroínas feministas herederas de las brujas del Medievo ("el feminismo espontáneo de la histeria" que dirá Emilce Dio). De esta forma, se ha interpretado la histeria y sus síntomas como la única forma posible de protesta o resistencia que tenían las mujeres frente a la represión victoriana, "como salida al atolladero del ser mujer" (Showalter, 1985; Dio Bleichmar, 1991). Para Chesler (1972) o Ussher (1991), la locura y el confinamiento psiquiátrico eran la expresión de la falta de poder de las mujeres y del intento sin éxito de rechazar y superar ese estado. "La mayor parte de las mujeres en psiquiátricos no están locas. Lo que llamamos locura

\footnotetext{
* Tras la muerte de Firestone, Susan Faludi (2013) escribió: si la esquizofrenia se había definido como una "metáfora amarga" de la "situación cultural" de las mujeres, las feministas radicales se habían propuesto cambiar precisamente eso. Las feministas de la segunda ola tenían la esperanza de aliviar su aislamiento a través del refugio de la "poderosa hermandad". Pero el movimiento colapsó y tristemente se repitieron historias de "derrota social": la soledad dolorosa, la pobreza, la falta de vivienda o la enfermedad mental.
} 
puede estar causado o exacerbado por la injusticia y la crueldad dentro de la familia y la sociedad" (Chesler, 1972). ¿Es la locura en sí misma resistencia feminista, o el precio a pagar por la misma? Las vidas de Millett o Firestone, con sus crisis, "parálisis emocionales" e internamientos tras duros conflictos familiares $^{5}$ y agotadoras luchas dentro del propio feminismo, nos hacen reflexionar sobre esta pregunta.

También en la década de los 70, diferentes académicas feministas criticaron públicamente "las violencias de género" en la práctica clínica psicológica. Phyllis Chesler en la conferencia anual de la APA en 1969 sorprendió a su audiencia demandando "un millón de dólares 'en reparaciones' para aquellas mujeres que nunca habían sido ayudadas por los profesionales de la salud mental y que en cambio sí habían sido objeto de abuso: etiquetadas negativamente, sedadas, seducidas sexualmente durante tratamiento, hospitalizadas contra su voluntad, objeto de descargas eléctricas, lobotomías, y sobre todo, rechazadas como demasiado 'agresivas', 'promiscuas', 'depresivas', 'feas', 'viejas', 'desagradables' o 'incurables'” (en Chesler, 1972). El acto fue cubierto por el New York Times" bajo el título "Women criticize psychology unit" donde se describía la estupefacción de una audiencia de 2000 miembros de la APA incapaces de tomarse en serio la crítica a una psicoterapia que había "perpetuado la dominación masculina y contribuido a la enfermedad mental en mujeres".

Producto de la rabia que le provocó dicho evento y sus compañeros de disciplina (como ella misma cuenta), Chesler (1972) publicó tres años después Women $\mathcal{E}$ Madness. En él por primera vez introducía la palabra "patriarcado" en la literatura psicológica. Denunció cómo las mujeres eran categorizadas como mentalmente inestables tanto si se conformaban a los dictados de la feminidad como si se rebelaban a ellos, y cómo los psicólogos y psiquiatras varones habían construido la locura y la feminidad de forma "especular": "la

\footnotetext{
${ }^{5}$ En el primer caso, con su madre y sectores del feminismo por declararse públicamente como lesbiana; en el segundo, con su padre, por romper con las normas de la mujer judía "correcta" y con sectores de las feministas radicales por su liderazgo. https: / www.nytimes.com/1970/09/06/archives/women-criticize-psychology-unit-1million-inreparations-is-demanded.html
} 
locura, tanto si aparece en hombres como en mujeres, es o bien la ejecución del rol femenino devaluado o el rechazo total o parcial de los estereotipos de rol de género" (Chesler, 1972). Siguiendo las investigaciones de Inge Broverman, criticaba el modelo androcéntrico y los dobles estándares en salud mental: la descripción de un adulto sano coincide con el estereotipo masculino, el estereotipo femenino ha sido visto como psicológicamente enfermo. Así, una mujer normal, sana y del promedio ha sido un ser humano loco-neurótico. Si la mujer sigue su rol de género adquiere características de personalidad indeseables (pasividad, dependencia, emotividad), pero si desarrolla rasgos humanos deseables pierde su feminidad (asertividad, independencia, sexualmente activa, etc.). Adrienne Rich (1972) hizo una brillante reseña al libro en el New York Times: "necesitamos saber más sobre las percepciones de las mujeres sobre sus estados psíquicos, los cuales han sido descritos por los analistas masculinos durante demasiado tiempo, ajenos a su propia subjetividad y a sus propios puntos ciegos".

El sexismo y androcentrismo en la práctica psicoterapéutica venía reforzado por la formación psicoanalítica (no precisamente en las críticas feministas de Karen Horney a Freud) y la aceptación acrítica de la teoría de la seducción de Freud, que conducía a desconfiar del relato de las mujeres por ejemplo en casos de abuso o violación (Marecek, 2017; Hure-Mustin, 2017). Juliet Mitchell (1974) o Luce Irigaray (1974) aportaron claves feministas para la crítica al psicoanálisis y su falogocentrismo desde dentro de la corriente. También los terapeutas familiares o sistémicos (incluidos los que venían de la antipsiquiatría como Cooper o Laing) conceptualizaban la salud familiar en términos de estereotipos de género y culpaban a las madres de los problemas de los niños -o bien las idealizaban- (Ussher, 1991; Hure-Mustin, 2017). Que no se salvaba ninguna escuela teórica lo muestran los vídeos que la $\mathrm{APA}^{8}$ creó para la formación de terapeutas en 1965 donde Gloria, así se llamaba la paciente, se enfrentaba a tres tipos de terapia: la racional emotiva de Ellis, la gestáltica de Pearls y la humanista de Rogers. Ninguna era capaz de identificar

\footnotetext{
https: / / www.nytimes.com / 1972 /12 /31 / archives / women-and-madness-by-phyllis-chesler-illustrated359-pp-new-york.html

s Los videos fueron publicados con el titulo de Three Approaches to Psychotherapy: A Film Series.
} 
y comprender los malestares de Gloria desde los mandatos sociales y de género (muchos contradictorios). Bajo un aparente modelo neutro de clientepaciente, los tres descuidaban las relaciones y dinámicas de poder desde un prisma puramente individualista y psicologicista. Y los tres enmarcaban el sufrimiento psicológico "dentro" de Gloria (por no aceptarse, por cogniciones disfuncionales, etc.), despolitizando e invisibilizando los efectos del patriarcado en la psique de las mujeres. Siguiendo a Greenspan (1983), en New approach to women $\mathcal{E}$ therapy, en los vídeos estaban presentes los mitos de la práctica terapéutica tradicional: el mito del reduccionismo intrapsíquico, el mito de la objetividad y distancia del experto varón, y el mito del modelo médico de los síntomas.

Por otro lado, en 1974 la psicoterapeuta feminista Rachel Hare-Mustin publicó un artículo que abordaba la falta de ética del "uso del contacto sexual en psicoterapia", en un momento en el que no pocos terapeutas (masculinos) consideraban que ofertar relaciones sexuales podría beneficiar a la paciente o lo justificaban apoyándose en que era la paciente la que les seducía y disfrutaba con ello'. El artículo mencionaba investigaciones previas que recogían estadísticas sobre este tipo de relaciones: todos los terapeutas eran varones en torno a los 40 y las clientes (salvo un caso) todas mujeres de 10 a 25 años menores que ellos. Prueba de la "normalización" de esta práctica fue la publicación de las sesiones de terapia de la escritora Anne Sexton por parte de su biógrafa, donde no pocas voces se alzaron criticando que no se había respetado la confidencialidad, pero el abuso sexual de su terapeuta no parecía sorprender (Hare-Mustin, 1992). La narrativa psicoanalítica y el modelo intrapsíquico dominantes no permitían ver la posición especial de poder del terapeuta y la de dependencia emocional de la paciente. Gracias a las presiones de las psicólogas feministas, se llevaron a cabo investigaciones sobre las consecuencias de esta práctica, muy parecidas a las de una violación (ira, sentimientos de culpa, flashbacks, indefensión, tristeza, problemas con los límites, etc.); y se consiguió que en 1977 la APA cambiara sus estándares éticos, prohibiendo explícitamente el sexo con pacientes.

\footnotetext{
' Hare-Mustin hace referencia a un panel en una de las convenciones de la APA de finales de los 60 donde cuatro terapeutas (varones) discutían sobre los pros y los contras de tener sexo con sus pacientes.
} 
El feminismo en alianza con la anti-psiquiatría denunció la autoridad del poder psicomédico sobre los cuerpos y vidas de las mujeres, y la patologización y atribución intra-psíquica (la psicologización) de conflictos subjetivos y malestares producto de la dominación masculina y heterosexual. Millett, Firestone o Chesler habían denunciado los psiquiátricos como estructuras que reproducían los guiones de la sociedad patriarcal, infantilizando a las mujeres y, sobre todo, ejerciendo violencia sobre ellas para que volvieran a su rol. Para muchas feministas, la psicoterapia era un agente del patriarcado, un "opio" para las mujeres; para Mary Daly (1979), representaba un tipo de "violación mental" donde se infectaba a las mujeres, no se las curaba. En la década de los 70, grupos feministas, en alianza con grupos de gays y lesbianas, organizaron protestas y boicots en convenciones de asociaciones psiquiátricas y psicológicas, denunciando la construcción social de enfermedades mentales a través de prejuicios sexistas, racistas, políticos y homófobos. Estos colectivos exigían el final de la culpabilización de las madres o de las víctimas de violencia sexual, la patologización de la agencia sexual en mujeres o de su ira; pero también protección legal frente a prácticas violentas y abusivas de clínicos y libertad para "prisioneros políticos" internados en instituciones mentales (ya fueran gays, lesbianas o mujeres que transgredían los roles de género). Como denunció Ussher (1991) en su libro Women's madness. Misoginy or mental illness?, el mayor número de terapias electroconvulsivas, psicocirugías o shock insulínicos se realizaba en mujeres. El ajuste al rol femenino era la medida de la salud mental de las mujeres y de su progreso psiquiátrico. Claramente el uso de dichas prácticas lo conseguía.

\section{LOS GRUPOS DE CONCIENCIACIÓN COMO HERRAMIENTAS FESMINISTAS PARA POLITIZAR “MALESTARES"}

Con este clima crítico, ¿cómo se podía luchar frente a la idea de la terapia feminista como un oxímoron?: a través de los grupos de concienciación o de toma de conciencia donde las mujeres hablaban de su propia opresión (Marecek, 2017). Katie Sarachild acuñó el término consciousness-raising como la práctica en pequeños grupos donde las mujeres reflexionaban sobre cómo 
experimentaban su opresión, para tomar conciencia de ella y generar acciones para transformarla. Los grupos fueron la marca del movimiento de liberación de las mujeres, al igual que su lema más conocido "lo personal es político" (Hanisch, 1969). Para el feminismo radical, la lucha política era básica para la salud mental; y los grupos de concienciación una herramienta fundamental para la transformación social. La liberación implicaba no solo cambiar instituciones sociales, también conciencias. Ante las críticas por parte de sectores de izquierda de que dichos grupos eran grupos de "autocontemplación", de "terapia personal" o "no políticos", y el rechazo que generaba llevar problemas personales (sobre el cuerpo, la sexualidad, etc.) al ámbito público, Carol Hanisch (1969) escribió “Lo personal es político". Hanisch argumentaba que no se trataba de "terapia", pues ésta supone que alguien está enfermo y que en ella está su cura (una solución personal). “Las mujeres tienen problemas, ¡no están enfermas! Lo que necesitamos es que cambien las condiciones objetivas, no ajustarnos a ellas y la terapia se está acomodando como alternativa al malestar personal". Hanisch aclaraba que en los grupos no se trataba de resolver problemas personales, porque los problemas personales son problemas políticos; tampoco de dar soluciones personales, sino de acción colectiva. Subrayaba también la dimensión epistémica de los grupos. Los saberes ("la explicación científica" en sus palabras) surgidos de la reflexión colectiva, de discutir las experiencias personales entre mujeres de forma horizontal, de analizarlas y buscar conexiones entre ellas y con su subordinación, han sido fundamentales para la teoría feminista. Y además, añadía, se pueden considerar los aspectos terapéuticos de los grupos (como "terapia política” en sus propios términos), por cuanto tienen el efecto de que las mujeres se deshacen de su culpa por sus fracasos u opresión. En 1983, Franca Basaglia señalaba a partir de su experiencia en mayo del 68, que la mujer, en lo privado, "paga el precio del compromiso político del hombre" (es la que "prepara la leche caliente de los revolucionarios"). Y añadía: con los grupos de concienciación del movimiento

\footnotetext{
${ }^{10}$ Si bien se suele atribuir a Kate Millett, el lema tiene más bien una autoría colectiva. Es el título de un texto de Carol Hanisch, pero como ella misma explicó, fue sugerido por las editoras del libro donde se publicó, en concreto por Sulamith Firestone y Anne Koedt. Todas ellas formaban parte del grupo de Feministas radicales de Nueva York.
} 
de liberación de la mujer, "lo privado había conquistado la dignidad de lo político" poniendo en discusión cada nivel de opresión.

Aunque muchas feministas situaban los grupos de toma de conciencia como el sustituto o antídoto político de la terapia, algunas terapeutas feministas tomaron los grupos de concienciación como modelos de una nueva forma de terapia (Marecek, 2017). Una terapia para la que el cambio personal (y los objetivos del tratamiento), la acción social y el cambio político estaban unidos, y donde identificar el sexismo y sus efectos en las biografías de las mujeres era un factor activo en el proceso de tratamiento. Para ello, el rol de la terapeuta tenía que ser más horizontal, menos autoritario y más facilitador (de "acompañamiento" que diríamos ahora). Se iniciaban así las bases de las "terapias no-sexistas" y "terapias feministas" (Hyde, 1995) o los grupos de mujeres creados y dirigidos por profesionales feministas que abordaban "malestares" desde el análisis social de las relaciones de poder sin patologizar a las mujeres (Sáez Buenaventura, 1988; Burin, 1990).

En la década de los 80, en el contexto español, Carmen Sáez Buenaventura editó Mujer, locura y feminismo donde se publicaba por primera vez en castellano el trabajo de Chesler o de otras terapeutas feministas como Pauline Bart, Rachel Hure-Mustin o Jeannet Marecek. Esta psiquiatra creó y organizó los “grupos de terapia de orientación feminista" ("para amas de cada con depresión"), descritos en su ya clásico Sobre mujer y salud mental (1988); Asunción González de Chávez (1993) también trabajó con "grupos terapéuticos de mujeres". En Argentina, los "talleres de reflexión" de mujeres como "agentes de salud" (de información, denuncia o concientización) fueron descritos por Mabel Burin, Esther Moncaraz y Susana Velázquez (1990) en otro libro fundamental El malestar de las mujeres. La tranquilidad recetada, donde abordaban el problema del mayor consumo de psicofármacos en mujeres. Clara Coria (1987), por su parte, coordinó "grupos de reflexión" donde se trabajaba la dependencia económica y la salud mental de las mujeres ("el sexo oculto del dinero"). En la fuente de estos grupos, Coria reconocía la influencia de los grupos operativos del psicólogo social Pichón Riviere, la concientización de Paulo Freire y los grupos de mujeres autogestivos estadounidenses. La práctica grupal consistía en 
indagar en un tema, explicitar tensiones y, como consecuencia, que emergiera la "conciencia de género" que implicaba desnaturalizar situaciones opresivas de las mujeres (González Oddera, 2018 ${ }^{11}$ ). Todas ellas eran iniciativas feministas unidas al movimiento de salud mental, y en todos los casos no se dejaba de insistir en que las condiciones de vida de las mujeres, en especial su vida cotidiana, repercutían de modo decisivo en sus modos de enfermar. Algo que además se producía atravesado por la clase social.

\section{LAS CRÍTICAS FEMINISTAS A LAS VIOLENCIAS DE LOS DUALISMOS EN PSICOLOGÍA}

En Marcar la diferencia. Psicología y construcción de los sexos, Rachel Hare-Mustin y Jeanne Marecek (1994) distinguían dos tipos de sesgos en psicología: los "sesgos alfa", o la exageración de las diferencias y la polarización de género; y los "sesgos beta", cuando las diferencias de género son minimizadas y se considera lo masculino como referente universal. Ambos sesgos eran abordados (respectivamente) en los libros The mismeasure of woman. Why women are not the better sex, the inferior sex, or the opposite sex ${ }^{12}$ de la psicóloga social Carol Tavris (1992) y Psicología de la mujer. La otra mitad de la experiencia humana de Janet Hyde (1995). Se trata de dos sesgos aparentemente paradójicos: si se rompe el androcentrismo, se marcan las diferencias y se corre el riesgo de fijarlas; y si no se marcan las diferencias para romper el dualismo, se corre el riesgo androcéntrico de que lo masculino quede como representante de lo genérico. Su manejo dependerá de las necesidades de cada contexto.

En apartados anteriores desarrollamos el sesgo androcéntrico en salud mental. En este apartado nos detendremos en la exageración de las diferencias y en las violencias de los dualismos de sexo/género/deseo. Ya hemos descrito cómo las pioneras criticaron la existencia de diferencias naturales en la mente de ambos sexos. Como consecuencia, la psicología abandonó la idea del "experimento perfecto" para encontrar las verdaderas diferencias cognitivas entre varones y mujeres (Morawski, 1988). Pero, tras la primera guerra mundial y la aplicación

\footnotetext{
"Tanto los grupos como los talleres de reflexión bebieron también del trabajo previo con grupos y con clara conciencia de género de Marie Langer.

${ }_{12}$ El título hace referencia al libro de Stephen Gould La falsa medida del hombre.
} 
de test de inteligencia en la selección de reclutas, la disciplina pasó a investigar las diferencias sexuales en inteligencia medidas a partir de dichas tecnologías. El que no se encontraran datos contundentes sobre las diferencias entre ellos y ellas, probablemente influyó en que comenzaran a estudiarse las diferencias en personalidad. Ello supuso pasar del modelo de inferioridad en inteligencia al modelo de complementariedad en personalidad -que camuflaba el anterior(Morawski, 1988).

Así, en la década de los 30, surge el primer test psicológico que medía masculinidad y feminidad (M/F), creado por Lewis Terman y Catharine Miles, con el objetivo claro de detectar de forma temprana "desviaciones sexuales" (varones femeninos y mujeres masculinas) para posteriormente tratarlos vía hormonal o modificación de conducta. Se iniciaba así una larga trayectoria dentro de la historia de la psicología de construcción de la masculinidad/feminidad (consciente o inconsciente) como rasgos de personalidad dentro del individuo, accesibles al experto mediante pruebas psicológicas (Morawski, 1985). También fue el punto de partida para detectar, regular y corregir cruces no alineados entre sexo y género confundidos con homosexualidad (en concreto, a la psicología le interesaban varones femeninos asimilados directamente a varones homosexuales). En la década de los 50 , se crea la Escala 5 de Masculinidad/Feminidad en el test de personalidad MMPI's. El hecho de que la dimensión de feminidad se validara con una muestra de 13 varones gays da cuenta de hasta qué punto la psicología del momento estaba obsesionada con las "desviaciones" (para modificarlas) y confundía género con preferencia sexual (Lewin, 1984).

Por su parte, la presencia de la homosexualidad en sucesivas versiones del DSM hasta 1973 demandaba la producción de dichas tecnologías de regulación de género. En 1975 la APA votó prohibir la discriminación frente a psicólogos gays y lesbianas que hasta dos años antes -1973- estaban etiquetados como enfermos mentales en el DSM por sus propios compañeros de profesión (Herman, 1994). En 1985 se establece la Sociedad para el "Estudio psicológico sobre cuestiones

${ }^{13}$ The Minnesota Multiphasic Personality Inventory ha sido y es uno de los test de personalidad más usados en psicología. 
de gays y lesbianas". Dichos cambios fueron posibles gracias a las protestas de activistas por la diversidad sexual en convenciones de la APA, denunciando la construcción de la homosexualidad como enfermedad mental y el uso del encierro, lobotomías y técnicas aversivas para curarla.

En la década de los 70, serán dos feministas, Anne Constantinople (1973) y Sandra Bem (1974) $)^{14}$, las que criticarán este modelo unidimensional de M/F con dos polos excluyentes alineados prescriptivamente con el dualismo sexual. Bem planteará un modelo de dos dimensiones, de mayor a menor feminidad y de mayor a menor masculinidad, donde cualquier persona se podía ubicar independientemente de su sexo (y propuso la "androginia psicológica" con partes combinadas de masculinidad y feminidad como referente saludable para ambos sexos). Constantinople, por su parte, directamente criticó la M/F como constructos psicológicos y aludió a su construcción social. Iniciaron así un cambio importante dentro de la psicología, se separaba la masculinidad/feminidad de la orientación sexual, pero además se dejaba de asociar la desviación o la patología a los cruces de sexo-género (mujeresmasculinas o varones-femeninos), y comenzaban a investigarse los efectos en salud mental de la conformidad con los roles de género.

Frente a los usos políticos, sociológicos o epistemológicos del concepto "género", la psicología tendía a entenderlo en términos de diferencias sexuales, rasgos de personalidad o como "identidad de género". La obsesión por los estudios sobre diferencias entre mujeres y varones en psicología, planteados como "estudios de género", llevó a algunas psicólogas feministas a denunciar que "la cuestión sobre las diferencias sexuales no era una cuestión feminista" (Unger, 2007:489). Las psicólogas feministas criticaron la teorización del género como rasgo diferencial y no como relaciones de poder, la reificación de conceptos como "masculinidad" y "feminidad", la construcción de una polarización dicotómica de los sexos y géneros con su consecuente heterosexismo, y el olvido de que el género está subjetiva y culturalmente situado (Bem, 1993; Hare-Mustin y Marecek, 1994). El nuevo neurosexismo, o

\footnotetext{
${ }^{14}$ La revista Feminism \& Psychology en su número 15 (año 2005) le dedicó un monográfico al artículo de Constantinople y sus implicaciones para la psicología. Por su parte, Sandra Bem (1993) escribió The lenses of gender donde rompía con su propuesta de androginia y criticaba la polarización de género
} 
neuro-obsesión más bien, con cerebros de hombre y de mujer, es quizá el exponente más popular y divulgado hoy en día de este sesgo reduccionista y esencializador.

Por otro lado, y recogiendo los orígenes clínicos del concepto "identidad de género" vía Robert Stoller o John Money, la disciplina se puso al servicio del diagnóstico de los "trastornos de la identidad de género" (TIG) o de la transexualidad ("curiosamente" cuando la homosexualidad salía del DSM, entraba el TIG). Los problemas aquí venían de un concepto de identidad de género que, aunque independiente del "sexo biológico", se fijaba de forma fija e irreversible, a modo de impronta, durante los primeros años de la infancia. Es decir, el paradigma de la identidad de género de los años 60 presuponía el dualismo sexual (solo existen dos sexos), la monosexualidad (cada persona solo puede tener un único sexo), pero también la existencia de una única, constante e irreversible identidad de género dentro del dualismo excluyente, así como la necesidad de coherencia sexo/génerodeseo. Identidades no binarias no tenían cabida.

Ello ha implicado, hasta nuestros días, la figura del psiquiatra o psicólogo como experto perito en el diagnóstico de identidad de género $\mathrm{y}$, con ello, de su "trastorno" (a veces confundiendo la masculinidad y feminidad medida por los test con el sexo sentido). También su papel como paso obligado en la detección de la "verdadera" transexual: aquella que necesariamente rechaza su cuerpo y sus genitales desde una narrativa del malestar o la disforia desde la infancia.

Lo que vinieron a aportar dos psicólogas sociales feministas, Suzanne Kessler y Wendy McKenna (1978), en Gender: An ethnomethodological approach ${ }^{15}$, es que la identidad no es solo una cuestión de psique o cuerpo, se construye en relación (la importancia de miradas, deseos y reconocimientos) y es un proceso cambiante. En este sorprendente trabajo, y anticipándose a las tesis teóricas de Judith Butler, las autoras rechazaban el dualismo sexo-biológico y génerosocial, al cuestionar la realidad "natural" e invariante de dos únicos sexos, "varón" o "mujer" - a los que se refieren como "géneros" por su carácter

${ }^{15}$ Feminism E Psychology dedicó en el 2000 un número monográfico a la revisión de este libro. 
también social. Buena parte del libro está dedicado a explicar cómo "se hace el género" en las interacciones cotidianas, mediante estrategias de presentación y passing -de formas de hablar, apariencia física pública y privada y la construcción de un pasado personal- y mediante atribuciones externas de género -comenzando por la primera asignación de sexo cuando nace un bebé.

La radicalidad de este libro -y de posteriores trabajos de Kessler sobre la asignación de sexo en bebés intersexuales- reside en tomar la dualidad sexual no sólo la de género- no como un apriori incuestionado sino como producto social. Por otro lado, al ubicar la identidad de género en un plano relacional y subjetivo, también performativo, anticipaban el grito político "la disforia no está en mi cuerpo, está en tu mirada", depositando el éxito del reconocimiento de la persona trans no tanto en su gestión corporal sino en el "tránsito" de quien mira.

\section{DE LO PERSONAL ES POLÍTICO A LO POLÍTICO ES PERSONAL: ¿LA PSICOLOGÍA RECONSTRUYE EL FEMINISMO?}

No pocas psicólogas feministas se han planteado el dilema "activismo versus academia" (Unger, 1998).

\footnotetext{
“Comprometidas con la práctica feminista somos excluidas de la categoría de 'psicólogas'. Practicando como 'psicólogas' dejamos de actuar como feministas (...) El híbrido 'psicología feminista' puede ser conceptualmente coherente o bien a través de una politización de la psicología, o bien a través de una despolitización del feminismo" (Kitzinger, 1990: 124 y 132).
}

Desde criterios empiristas de objetividad y neutralidad, parece complicado teorizar complejos mecanismos de opresión. Tampoco resulta fácil el engarce feminista entre la activista y la profesional de la salud (ya no digamos si ejerce desde la terapia individual). 
Sue Wilkinson (1991) ha señalado la asimetría de la relación entre la psicología y el feminismo: ¿qué tiene la psicología tal y como está definida que ofrecer a las feministas? La autora señala la incompatibilidad de valores y objetivos entre el feminismo y la psicología y los peligros de co-optación y desinflamiento político que puede implicar el encuentro entre ambos. Sabemos que cuando ha sido a través de la salud de las mujeres o diferencias de sexo/género, sin una crítica al positivismo, al individualismo y al esencialismo, ha implicado la despolitización del feminismo.

Por otro lado, si el concepto género fue una categoría de análisis útil, es necesario revisar sus excesos y derivas académicas e institucionales (también los de la "violencia de género"), en particular, aquellos que terminan despolitizándolo y psicologizándolo (o, “cerebrizándolo"). Es necesario politizar las tensiones entre "marcar las diferencias" de género (para denunciar desigualdades) y no reproducir los dualismos o invisibilizar las opresiones que intersectan. También revisar discursos psicológicos que parten de igualdades entre los sexos ya conseguidas (por ejemplo, sobre violencias bi-direccionales sin atender a las relaciones de poder o privilegios) o de decisiones bajo condiciones de "libertad individual" sin atender a las constricciones sociales.

"Lo personal es político" de la política feminista, donde los detalles personales de la vida eran objeto de discusión política, está dando paso a "lo personal es político" de la psicología, que implica que asuntos políticos son reducidos a cuestiones psicológicas individuales. Se mantiene así lo personal en personal y lo político como personal también (Kitzinger y Perkins, 1993). La introducción y aceptación de constructos psicológicos en el vocabulario político es prueba de ello: conceptos como empoderamiento, resiliencia o buena parte del lenguaje emocional y "yoico". La necesidad de teorizar los mecanismos psíquicos del 
poder no puede llevar a teorizar el poder o las opresiones en términos individualizados y privatizados (como si la revolución o la liberación tuvieran que venir desde dentro de la persona). El activismo no puede ser sustituido por una movilización cuyo protagonista sea un yo-emocional.

Hemos pasado de politizar la salud (la salud es política) a sanitarizar -y con ello psicologizar e individualizar- lo político. Cada vez más conflictos sociales o políticos terminan en la consulta individual (siempre y cuando se tenga el suficiente poder adquisitivo) o en los psicofármacos. Por otro lado, hasta el DSM ha co-optado el propio concepto de malestar: su quinta edición está llena de "malestares clínicamente significativos" que aunque sean producto de desigualdades sociales se conceptualizan como trastornos (la transexualidad es un claro ejemplo de ello).

¿Es ahora la psicología, en complicidad con discursos y prácticas neoliberales, la que intenta reconstruir el feminismo? Para evitarlo y mantener la perspectiva crítica, sigue siendo necesario reconocer el papel de los movimientos sociales y los activismos en la generación de conocimiento crítico y la transformación y revisión de las disciplinas.

\section{BIBLIOGRAFÍA}

Basaglia, Franca (1983). Mujer, locura y sociedad. Puebla: Benemérita Universidad Autónoma.

Bem, Sandra (1974). The measurement of psychological androgyny. Journal of Consulting and Clinical Psychology, 42(2), 155-162.

Bem, Sandra (1993). The Lenses of Gender. Transforming the Debate on Sexual Inequality. New Haven: Yale University Press.

Bohan, Janis S. (1992). Re-placing Women in Psychology. Iowa: Kendall/Hunt Publishing Company. 
Burin, Mabel; Moncaraz, Esther y Velázquez, Susana (1990). El malestar de las mujeres: La tranquilidad recetada, Paidós: Buenos Aires.

Burman, Erica (1998). La Deconstrucción de la Psicología Evolutiva. Madrid: Visor.

Calkins, Mary Whiton (1896). Community of ideas of men and women. Psychological Review, 3(4), 426-430.

Constantinople, Anne (1973/2005). Masculinity-Femininity: An Exception to a Famous Dictum?. Feminism \& Psychology, 15, 385-407.

Coria, Clara (1987). Grupos de reflexión, dependencia económica y salud mental de las mujeres. En Mabel Burin (ed.), Estudios sobre la subjetividad femenina: mujeres y salud mental (233-262). Buenos Aires: Librería de mujeres.

Chesler, Phyllis (1972). Women and Madness. New York: Doubleday.

Daly, Mary (1979). Gyn/Ecology: The Metaethics of Radical Feminism. New York: Beacon Press

Dio Bleichmar, Dio (1991). El feminismo espontáneo de la histeria. Madrid: Siglo XXI.

Ehreinreich, Barbara y Deirdre English (1990). Por su propio bien. Madrid: Taurus.

Faludi, Susan (2013, 15 abril). Death of revolutionary. The New Yorker. https: / / www.newyorker.com/magazine/2013/04/15/ death-of-arevolutionary

Firestone, Sulamith (1970). La dialéctica del sexo. Barcelona: Kairós.

Firestone, Sulamith (1998). Airless Spaces. Pasadena: Semiotext(e).

Flecha, Consuelo (1996). Las primeras universitarias en España. Madrid: Narcea.

Friedan, Betty (1963). La mística de la feminidad. Madrid: Júcar.

García Colmenares, Carmen (2011). Las primeras psicólogas españolas. Granada: Feminae.

García Dauder, Silvia (2005). Psicología y Feminismo. Madrid: Narcea.

García Dauder, (S.) (2010). La historia olvidada de las mujeres de la Escuela de Chicago. Reis, 131, 11-41.

González de Chávez, Asunción (comp.) (1993). Grupos terapéuticos de mujeres. En Cuerpo y subjetividad femenina (279-288). Madrid: Siglo XXI:

González Oddera, (2018). La subjetividad femenina en cuestión. Psicología y estudios de la mujer en la Argentina. Estudios de género, 4. https: / / estudiosdegenero.colmex.mx/index.php/eg/article/view/199 $/ \mathrm{pdf}$ 
Greenspan, Miriam (1993). A new approach to women $\mathcal{E}$ therapy. New York: Tab Books.

Guevara Ruiseñor, Elsa (2015). Ellas cambiaron la psicología. México: UNAM.

Hanisch, Carol (1969). Lo personal es político. Disponible en: http: / / www.diariofemenino.com.ar/documentos / lo-personal-espolitico_final.pdf

Harding, Sandra (1991). Whose Science? Whose Knowledge? Buckingham: Open University Press.

Harding, Sandra (1996). Ciencia y feminismo. Madrid: Morata.

Harding, Sandra (2015). Objectivity E Diversity. Chicago: University of Chicago Press.

Hare-Mustin, Rachel (1974). Ethical considerations in the use of sexual contact in psychotherapy. Psychotherapy: Theory, Research \& Practice, 11(4), 308310.

Hare-Mustin, Rachel (1992). Cries and whispers: The psychotherapy of Anne Sexton. Psychotherapy: Theory, Research, Practice, Training, 29(3), 406-409.

Hare-Mustin, Rachel (2017). Those Were the Best of Times, and Then.... Women E Therapy, 40(3-4), 346-357.

Hare-Mustin, Rachel y Marecek, Jeanne (1994). Marcar la diferencia. Psicología y construcción de los sexos. Barcelona: Herder.

Herman, Ellen (1994). Psychiatry, Psychology, and Homosexuality. New York: Chelsea House Publishers.

Herman, Ellen (1995). The romance of American Psychology. Berkeley: University of California Press.

Hollingworth, Leta Stteter (1914). Functional Periodicity: An Experimental Study of the Mental and Motor Abilities of Women during Menstruation. New York: Columbia University Press.

hooks, bell (1984). Feminist Theory. From margin to center. Boston: South end press.

Hyde, Janet S. (1995). Psicología de la mujer. La otra mitad de la experiencia humana. Madrid: Morata.

Irigaray, Luce (1974). Speculum: espéculo de la otra mujer. Madrid: Saltés.

Kitzinger, Celia (1990). Resisting the Discipline. En Erica Burman (ed.), Feminists and Psychological Practice (119-134). London: Sage.

Kitzinger, Celia (1993). "Psychology Constructs the Female": A Reappraisal. Feminism \& Psychology, 3(2), 189-193. 
Kitzinger, Celia y Perkins, Rachel (1993). Changing our minds. Lesbian feminism and psychology. London: Only Women Press.

Lerner, Gerda (1992). Placing Women in History: Definitions and Challenges. Re-placing Women in Psychology. Iowa: Kendall/Hunt Publishing Company.

Lewin, Miriam (1984). Psychology Measures Femininity and Masculinity, 2. En Miriam Lewin (ed.), In the Shadow of the Past: Psychology Portrays the Sexes (179-204). New York: Columbia University Press.

Marecek, Jeanne (2017). Blowing in the Wind:'70s Questions for Millennial Therapists. Women E Therapy, 40(3-4), 406-417

Millett, Kate (1969). Política Sexual. Madrid: Cátedra.

Millett, Kate (1974). En pleno vuelo. Barcelona: Hacer Editorial.

Millett, Kate (1990). The Loony-Bin Trip. Chicago: University of Illinois Press.

Mitchell, Juliet (1974). Psicoanálisis y feminismo. Barcelona: Anagrama.

Morawski, Jill (1985). The measurement of masculinity and femininity: Engendering categorical realities. Journal of Personality, 53(2), 196-223.

Morawski, Jill (1988). The rise of experimentation in American Psychology. New Haven: Yale University Press.

Morawski, Jill (1997). Practicing Feminisms, Reconstructing Psychology. Michigan: The University of Michigan Press.

Nogueiras, Belén (2018). La teoría feminista aplicada al ámbito de la salud de las mujeres. Tesis doctoral. Madrid, UCM.

Riviere, Joan (1929/1986). Womanliness as a Masquerade. En Victor Burgin, James Donald y Cora Kaplan (eds.), Formations of Fantasy (35-44). London: Methuen.

Rosenberg, Rosalind (1982). Beyond separate spheres: Intellectual roots of modern feminism. New Haven: Yale University Press.

Rossiter, Margaret (1992). Women Scientists in America. London: The Johns Hopkins University Press.

Sáez de Buenaventura, Carmen (1988). Mujer y salud mental. Barcelona: La Sal.

Sáez de Buenaventura, Carmen (ed.). (1979). Mujer, locura y feminismo. Barcelona: Dédalo.

Sarachild, Kathie (1978). Consciousness raising: a radical weapon. Disponible en: https: / / www.rapereliefshelter.bc.ca/ sites/default/files/imce/Feminis t-Revolution-Consciousness-Raising--A-Radical-Weapon-KathieSarachild.pdf 
Scarborough, Elizabeth y Laurel Furumoto (1987). Untold Lives: The First Generation of American Women Psychologists. Nueva York: Columbia University Press.

Sherif, Carolyn Wood (1979). What every intelligent person should know about Psychology and Women. En Eloise Snyder (ed.), The Study of Women: Enlarging perspectives of social reality_(143-183). New York: Harper \& Row.

Sherif, Carolyn Wood (1979/1987). Ethnocentrism, Androcentism, and Sexist Bias in Psychology. En Sandra Harding (ed.), Feminism and Methodology (39-96). Bloomington: Indiana University Press.

Showalter, Elaine (1985). The Female Malady. London: Virago.

Taft, Jessie (1916). The woman's movement from the point of view of social consciousness. Chicago: University of Chicago Press.

Tanner, Amy (1896). Community of ideas of men and women. Psychological Review, 3(5), 548-550.

Tavris, Carol (1992). The mismeasure of woman. New York: Simon \& Schuster.

Tuana, Nancy (2006). The speculum of ignorance: The women's health movement and epistemologies of ignorance. Hypatia, 21(3), 1-19.

Unger, Rhoda (1979). Toward a redefinition of sex and gender. American Psychologist, 34(11), 1085-1094.

Unger, Rhoda (1998). Resisting Gender. Twenty-five years of Feminist Psychology. London: Sage.

Ussher, Jane (1991). Women's Madness. Amherst: The University of Massachusetts Press.

Ussher, Jane (2013): Diagnosing difficult women and pathologising femininity: Gender bias in psychiatric nosology. Feminism \& Psychology, 23(1), 6369.

Vertinsky, Patricia (1994). The eternally wounded woman. Women, doctors and exercise. Illinois: University of Illinois Press.

Weisstein, Naomi (1968/1993). Psychology Constructs the Female. Feminism $\mathcal{E}$ Psychology, 3(2): 195-210.

Wilkinson, Sue (1991). Why Psychology (Badly) Needs Feminism? En Jane Aaron y Sylvia Walby (eds.), Out of the Margins (191-203). London: The Falmer Press.

Winkler, Inés (2007). Pioneras sin monumentos. Mujeres en Psicología. Santiago de Chile: LOM.

Woolley, Helen Thompson (1903). The Mental Traits of Sex. Chicago: Univ. of Chicago Press. 
Woolley, Helen Thompson (1910). Psychological Literature. A review of the recent literature on the psychology of sex. The Psychological Bulletin, 7, 335-342. 